\title{
INTER-CELL LOAD BALANCING TECHNIQUE FOR MULTI-CLASS TRAFFIC IN MIMO-LTE-A NETWORKS
}

\author{
${ }^{1}$ T.PADMAPRIYA, ${ }^{2} \mathrm{~V}$. SAMINADAN \\ ${ }^{1}$ Research Scholar, Pondicherry Engineering College \\ ${ }^{2}$ Professor, Department of ECE, Pondicherry Engineering College \\ E-mail: ${ }^{1}$ padmapriyat1085@gmail.com
}

\begin{abstract}
In LTE network, load imbalance is the crucial issue which needs to be handled in order to exploit most of the benefit from LTE without degrading the throughput. In this paper, we have proposed an Inter-cell Load Balancing Technique for Multi-class Traffic in MIMO-LTE-A Networks. First, we have proposed a robust load balancing framework to efficiently handle the traffic and also to keep the throughput as high as possible. To detect the overloaded cell, Call Blocking Ratio (CBR) is used as triggering mechanism. Moreover, to efficiently balance the congested cell detected by CBR Heaviest-First-Load-Balancing algorithm is implemented to avoid congestion in the traffic.
\end{abstract}

\section{INTRODUCTION}

\section{A. MIMO-LTE Networks}

In order to cope with the increased traffic demand, the 3rd generation partnership project (3GPP) has developed the Long Term Evolution (LTE) standard for $4 \mathrm{G}$ cellular networks. This is based on Orthogonal Frequency Domain Multiplexing (OFDM) waveform for downlink (DL) and Single Carrier Frequency Domain Multiplexing (SC-FDM) waveform for uplink (UL) communications. The key objectives of LTE networks are user high data rates, reduced latency, improved system capacity and coverage, low complexity, reduced cost of operation and seamless integration with existing systems [1] [2].

3GPP LTE networks can achieve high spectrum efficiency due to the usage of multi-input and multioutput (MIMO) antenna and orthogonal frequency division multiple (OFDM) technology. There are many MIMO schemes standardized in 3GPP systems, and the base station scheduler has the capability to optimally select the MIMO scheme that suits the channel conditions of the mobile. A fundamental MIMO scheme is that of precoded spatial multiplexing (SM) where multiple information "streams" are transmitted simultaneously from the base station to the mobile. These techniques are appropriate in high SINR areas with rich scattering environments, in combination with suitable antenna configurations. However, the network performance is still influenced by several factors, among which intercell interference (ICI) and load imbalance are two major ones [3] [5].

\section{B. Load balancing in LTE Networks}

Load Balancing is defined as an automatic way to resolve the overloading by shifting traffic towards the light-loaded cells, by making use of the radio resources more efficiently across the whole network. One possible way to balance the network load is to adjust the network control parameters in such a way that overloaded cells can offload the excess traffic to low-loaded adjacent cells, whenever available. However, this action might introduce additional handovers, which might cause bad handover performance, leading to the result that system would adjust handover parameters to reorganize the situation, which might be in contradiction to the aim of load balancing [4].

Load balancing scheme is required to minimize the demanded radio resources of the maximum loaded cell to avoid the traffic congestion in long term evolution (LTE) networks. Load imbalance in LTE networks deteriorates the system performance influenced by unbalanced load distribution among nearby cells. Hence real-time inter-cell optimization is adaptable to environment especially when unbalanced and time varying, is needed [6] [7] [10].

Issues

- Handover failure

- Load distribution

- Signaling overhead

- Congestion

- Radio resources [8] [10] [11]

\section{LITERATURE REVIEW}

Wenyu LI et al [4] have proposed a dynamic hysteresis-adjusting. With this proposed method, the two SON aspects load balancing and handover parameter optimization can achieve a better coordination. The new method tunes the hysteresis according to a key indicator radio link failure ratio, with realistic consideration, thus avoiding the possibility that load balancing has a bad influence on the network performance, for example, causing a higher radio link failure ratio and risk of jeopardizing the normal function of a network. With the proposed method, which is simple and easy to realize, the network handover performance and load balancing 
effect are both guaranteed compared with conventional solutions. However there occurs handover failure.

Hao Wang et al [6] have proposed network structure constraints and a practical suboptimal algorithm, called Heaviest-First Load Balancing (HFLB). Using the HFLB algorithm the network can get significantly better load balancing while maintaining the same network throughput at the price of a bit more handovers compared with the traditional signal strength-based handover algorithm. However the load balance index in mobile scenario is lower. And the radio resource consumption increases.

WANG Min et al [7] have proposed a min-max load balancing (LB) scheme to minimize the demanded radio resources of the maximum loaded cell. For the mixed multicast and unicast services, multicast services are transmitted by single frequency network (SFN) mode and unicast services are delivered with point-to-point (PTP) mode. The min-max LB takes into account point-to-multipoint (PTM) mode for multicast services and selects the proper transmission mode between SFN and PTM for each multicast service to minimize the demanded radio resources of the maximum loaded cell. Based on the solution of this minimization problem, if the maximum loaded cell does not overload, the min-max LB will change PTM mode into SFN mode for multicast services to achieve high quality of service (QoS). The proposed min-max LB scheme requires less radio resources from the maximum loaded cell than SFN mode for all multicast services.

LI Bo et al [8] have proposed an inter-domain cooperative traffic balancing scheme focusing on reducing the effective resource cost and mitigating the co-channel interference in multi-domain Het-Net. The detailed implementation for the proposed traffic balancing scheme is designed. In the numerical evaluation, the genetic algorithm (GA) as an optimization method is used to demonstrate that the total effective resource cost is significantly reduced through their proposed inter-domain traffic balancing scheme comparing with the intra-domain traffic balancing scheme. The $43 \%$ of the resource cost is saved. The proposed scheme has great advantages in interference management in Het-Net. However the cell-edge throughput and the average cell throughput is not increased effectively.

WANG Hao et al [9] have proposed a mobility load balancing (MLB) as an important use case in 3GPP self-organizing network (SON), in which the serving cell of a user can be selected to achieve load balancing rather than act as the cell with the maximum received power. In this paper, a unified algorithm is proposed for MLB in the LTE network. The proposed algorithm is evaluated for users with different kinds of QoS requirements, i.e., guaranteed bit rate (GBR) users with the objective function of load balance index and non-GBR (nGBR) users with the objective function of total utility, respectively.
The proposed algorithm leads to significantly balanced load distribution for GBR users to decrease the new call blocking rate, and for nGBR users to improve the cell-edge throughput at the cost of only slight deterioration of total throughput. However with the larger arrival rates the more will be unbalanced loading.

Ming Li et al [10] have proposed an LTE virtualization framework (that enables spectrum sharing) and a dynamic load balancing scheme for multi-eNB and multi-VO (Virtual Operator) systems. They compare the performance gain of both schemes for different applications, e.g. VoIP, video, HTTP and FTP. They also investigate the parameterization of both schemes, e.g. sharing intervals, LB intervals and safety margins, in order to find the optimal parameter settings. The LTE networks can benefit from both $\mathrm{NV}$ and LB techniques.

Omar Altrad et al [11] have proposed a general loadbalancing algorithm to help congested cells handle traffic dynamically. The algorithm is based on clustering methods and can be applied to any wireless technology such as LTE, WiMAX and GSM. The algorithm can be automatically controlled and triggered when needed for any cell on the system. It can be implemented in a distributed or semidistributed fashion. The triggering cycle for this algorithm is left for the operator to decide on; the underlying variations are slow so there is no need for fast self-optimizing network (SON) algorithms. The distribution of the load of the congested cell to its neighbor is one step only, which significantly reduces the signaling overhead and wasting of resources in the lightly-loaded cells compared to conventional methods.

\section{PROPOSED SOLUTION}

\section{A. Overview}

From the existing works, we can observe certain drawbacks, the handover failures in DHA [4], the load balance index in case of mobile scenarios decreases [6], the waste radio resources is saved for the unnecessary multicast services [7], due to periodic data collection [8] leads to large-scale signal changes and signaling overhead, the handover [10] might cause packet loss problem, the reduction of congestion [11] is almost less.

In this paper, we propose to design a load balancing framework for MIMO-LTE networks. A load balancing framework is developed [6] which balances the entire network load while keeping the network throughput as high as possible. Here by analyzing the complexity of the optimization problem, network structure constraints are presented, and a practical suboptimal algorithm called Heaviest-First Load Balancing (HFLB) is proposed. The main objective of this framework is to make use of enforced handover to balance the load between different cells and keep the network throughput as high as possible at the 
same time.For the detection of overloaded cells, call blocking ratio (CBR) [11] is used as the triggering method. Then the algorithm based on load balancing is invoked into the congested cells [6].

\section{B. Detection of Overloaded Cell}

In order to detect the overloaded cells, Call Blocking Ratio (CBR) is the real parameter which indicates the degradation of the system when any overload occurs as shown in Fig.2. Here, $\mathrm{m}$ is detected as the overloaded cell. In our proposed solution, CBR is used as a triggering mechanism to enhance load balancing in LTE:

\section{$\mathrm{CBR} \geq \tau$}

Where $\mathrm{CBR}=$ blocked calls/total accepted calls

Also, $\tau$ is predefined threshold reserved for operator use which is decided by the quality of service (QoS) achieved by transmission mode of multicast services.
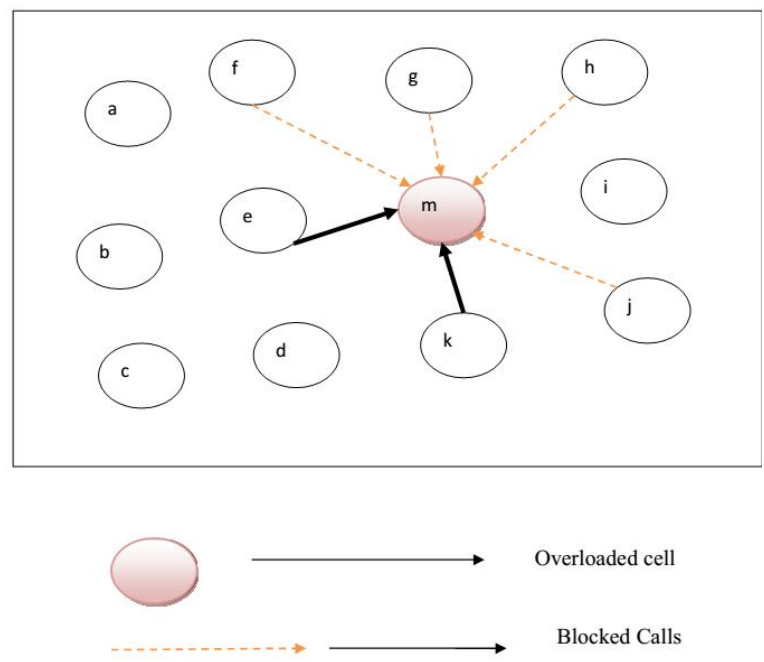

Figure 1. Detection of Overloaded Cell

\section{Inter Cell Load Balancing Using Heaviest First Load Balancing}

This section first describes about the detection of overloaded cell and the sub optimal algorithm that efficiently balance the load without affecting the throughput.

\section{a) Definition of Utility Function}

Given $|E|$ eNodeBs and $|N|$ mobile users, we first find an optimal assignment between mobile users and cells.

For this, first we define a utility function in the multicell network which is given as below:

$$
\mathrm{V}(\phi, \varphi)(\mathrm{t})=\phi \mathrm{P}(\mathrm{t})-\varphi \mu(\mathrm{t})
$$

Where $\phi \geq 0$ and $\varphi \geq 0$ are weighting coefficients on network throughput and load balance index respectively.

Different values of $\phi$ and $\varphi$ in solving the joint optimization problem in equation (1) can suitably be selected between the tradeoff between load balancing and network throughput.
Since, $\mathrm{P}(\mathrm{t})$ and $\mu(\mathrm{t})$ are both determined by the allocation between users and cells, the problem is to find the optimal allocation that maximizes ${ }^{\mathrm{V}(\mathrm{t})}$ for the current timeslot $t$.

Let load at each cell $i$ at time slot $t$ is given by

$$
\mathrm{L}_{\mathrm{i}}(\mathrm{t})=\mathrm{bu}(\mathrm{t}) / \mathrm{b}(\mathrm{t})
$$

where $b(t)$ and bu(t) denotes the number of PRB and number of used PRB at cell $i$.

The average load of the network at time $t$ is given by

$$
\mathrm{L1}_{\mathrm{i}}(\mathrm{t})=\sum_{i \in N} \operatorname{Li}(t) /|\mathrm{N}|
$$

Then load balance index ${ }^{\mu(\mathrm{t})}$ can be given by

$$
\mu(t)=\sum_{i \in N}(L i(t)-L 1 i(t))^{2}
$$

Define an allocation indicator variable $I_{m, n}(t)$, which is equal to 1 when eNodeB $m$ allocates a physical resource block (PRB) to user $n$ at timeslot $t$ or to 0 otherwise. Hence the load definition of cell $\mathrm{m}$ can be formulated as:

$$
\eta_{m}(t)=\sum_{n \in N} I_{m, n}(t) / b
$$

Here $b$ is the total number of PRB.

Representing the allocation by the matrix $I(t)=\left(I_{m, n}(t): m \in E, n \in N, \forall t \geq 0\right)$, hence the problem is equivalent to the following maximization problem with $\mathrm{I}(\mathrm{t})$ :

$$
\begin{aligned}
& \max _{I(t)} V(I, \phi, \varphi)(t)=\phi P(I(t))-\varphi \mu(I(t)) \\
& \text { s.t } \sum_{n \in N} I_{m, n}(t) \leq b, \forall m \in N \\
& \sum_{m \in N} I_{m, n}(t)=1, \forall n \in N, \\
& \sum_{m \in N} I_{m, n}(t) p_{m, n}(t) \geq \theta, \forall n \in N
\end{aligned}
$$

Where $P(I(t))=\sum_{n \in N} \sum_{m \in N} p_{m, n}(t) I_{m, n}(t)$ is the network throughput at the timeslot $t$.

$P_{m, n}(t)$ is the available Shanon rate at time slot $t$ given by

$$
\mathrm{P}_{\mathrm{m}, \mathrm{n}}(\mathrm{t})=\mathrm{W}_{\mathrm{i}, \mathrm{k}} \log _{2}+\mathrm{SNR}_{\mathrm{i}, \mathrm{k}}(\mathrm{t})
$$

Also, $\theta$ represents minimal throughput of each user.

The constraints in equation (7) represents that all cells have almost the same capacity limitation, and also the number of user operated by one eNodeB can't exceed the number of its total PRBs.

Constraint in equation (8) represents that one user can only be operated by one eNodeB at some specific timeslot $\mathrm{t}$.

Constraint in equation (9) represents that user can be operated by the eNodeB which can afford it a throughput value larger than the $\theta$

Assuming that cell $\mathrm{i}$ handovers a user $\mathrm{k}$ to a target cell $\mathrm{j}$ for load balancing, the following condition should be satisfied:

$$
\mathrm{u}(\mathrm{j})^{\prime}+\mathrm{u}(\mathrm{i})^{\prime}>\mathrm{u}(\mathrm{j})+\mathrm{u}(\mathrm{i})
$$


where u(j)' and u(i)' are the updated values of individual utility functions after handover for cell $\mathrm{j}$ and $\mathrm{i}$, respectively.

b) Heaviest First Load Balancing Algorithm

In each load balancing choose the heaviest loaded one whose load exceeds the threshold $\tau$ to perform load balancing according to Heaviest-First Load Balancing which is described as below:

//At the mth load balancing cycle//

1. Each and every eNodeBs receive load status from its neighboring cells with $C B R$.

2. Cell $m$ is the heaviest one.

3. if load of cell $m$ exceeds threshold $\tau$, go to next step

4. else stop.

5. In cell $m$, find user $n$ and target cell $c$ with the largest $y_{m, c}^{n}$.

6. If it satisfies inequality (11), then switch user $n$ to cell $c$

7. Update other users' gain in cell $m$, then go to next step

8. Else stop.

9. If load status of cell $m$ still exceeds threshold $\tau$,

10. then go to step 5.

10. Else stop.

\section{SIMULATION RESULTS}

\section{A. Simulation Model and Parameters}

The Network Simulator (NS2) [12], is used to simulate the proposed architecture. In the simulation, 50 mobile nodes move in a 1200 meter x 1200 meter region for 50 seconds of simulation time. All nodes have the same transmission range of 250 meters. The simulated traffic is Constant Bit Rate (CBR). The simulation topology is shows in Figure 4.

The simulation settings and parameters are summarized in Table- 1 .

Table-1 Simulation Settings

\begin{tabular}{|c|c|}
\hline No. of Nodes & 31 \\
\hline Area Size & $1200 \times 1200$ \\
\hline Mac & IEEE 802.11 \\
\hline Transmission Range & $250 \mathrm{~m}$ \\
\hline Simulation Time & $50 \mathrm{sec}$ \\
\hline Traffic Source & CBR \\
\hline Packet Size & 512 \\
\hline Rate & $1,1.5,2,2.5$ and \\
& $3 \mathrm{Mb}$ \\
\hline Initial Energy & $4.1 \mathrm{~J}$ \\
\hline Transmission Power & 0.660 \\
\hline Receiving Power & 0.395 \\
\hline Idle Power & 0.035 \\
\hline
\end{tabular}

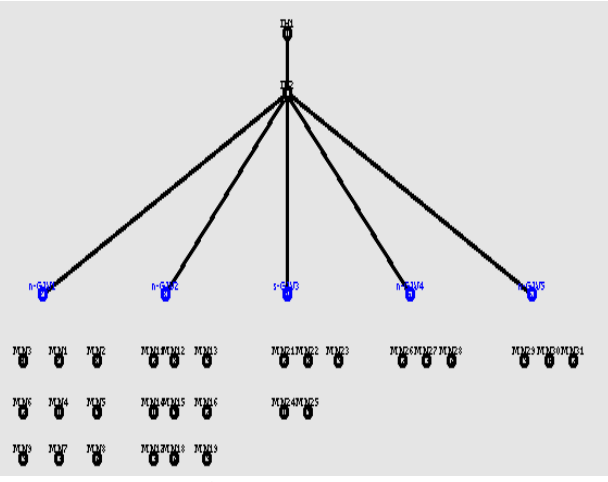

Figure 2: Simulation Topology

\section{B. Performance Metrics}

The proposed Distributed Load Balancing for Multiuser Multi-class Traffic (DLBMM) is compared with the HFLB technique [6]. The performance is evaluated mainly, according to the following metrics.

- Packet Delivery Ratio: It is the ratio between the number of packets received and the number of packets sent.

- Packet Drop: It refers the average number of packets dropped during the transmission

- Bandwidth Utilization: It is the amount of bandwidth utilized at the cell

- Delay: It is the amount of time taken by the nodes to transmit the data packets.

\section{Results}

Here the load of cell-1 is balanced among the cells 3 and 4. The transmission rate is varied as 1, 1.5, 2, 2.5 and $3 \mathrm{Mb}$ and the above performance metrics are evaluated at cell-1 for downlink CBR traffic. Figure 3 to 5 show the graphical representation of the results for Bandwidth utilization, delay, packet delivery ratio and packet drop.

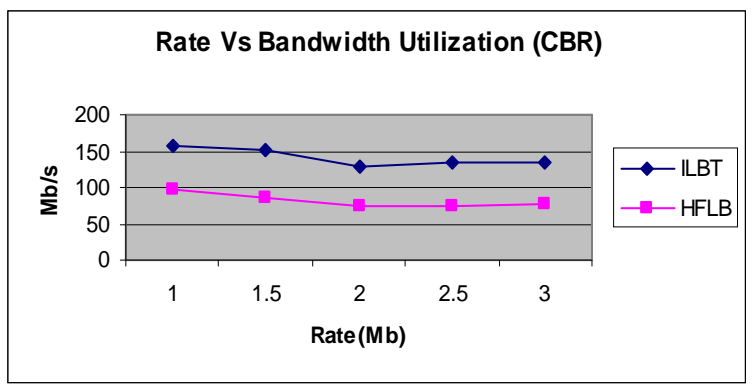

Fig 3: Rate Vs Received Bandwidth

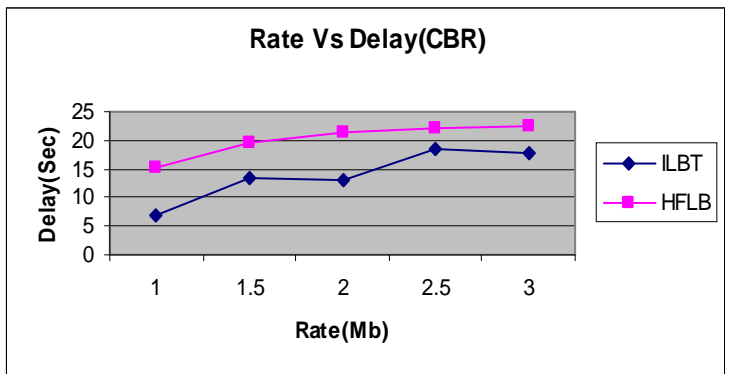

Fig 4: Rate VS Delay 


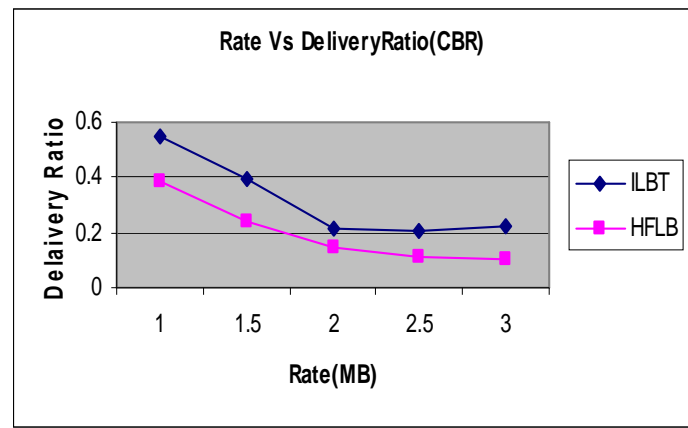

Fig 5: Rate Vs Delivery Ratio

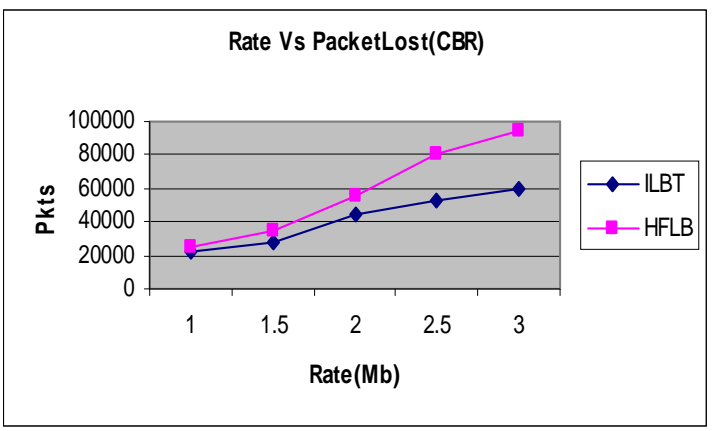

Fig 6: Rate Vs Packet Lost

Because of the distributed load balancing and optimization in ILBT, the overloaded traffic is evenly distributed, there by reducing the packet drop and improving the bandwidth utilization. Hence from the figures, we can see that ILBT outperforms HFLB in terms of bandwidth utilization by $42 \%$, delay by $33 \%$, packet delivery ratio by $39 \%$ and packet drop by $21 \%$.

\section{CONCLUSION}

In this paper, we have proposed a Distributed Load Balancing for Multi-user Multi-class Traffic in MIMO-LTE Networks. A robust load balancing framework is implemented that efficiently handles the overloaded traffic and also keep the throughput as high as possible. CBR is used to detect the overloaded cell that detects the load as soon as the service degradation happens. In order to efficiently handle the congested cell detected by CBR, HeaviestFirst-Load-Balancing algorithm is implemented to avoid any any kind of congestion in the network.

\section{REFERENCES}

[1] Madhavan Vajapeyam, Aleksandar Damnjanovic, Juan Montojo, Tingfang Ji, Yongbin Wei and Durga Malladi, "Downlink FTP Performance of Heterogeneous Networks for LTE-Advanced", Communications Workshops (ICC), 2011 IEEE International Conference on IEEE 2011.

[2] Ronoh Kennedy and Awoke Mengistie, "Load Balancing in Heterogeneous LTE-A Networks", (2012).

[3] Zhihang Li, Hao Wang, Zhiwen Pan, Nan Liu and Xiaohu You, "Dynamic Load Balancing in 3GPP LTE Multi-Cell Fractional Frequency Reuse Networks", Vehicular Technology Conference (VTC Fall), 2012 IEEE 2012.

[4] Wenyu LI, Xiaoyu DUAN, Shucong JIA, Lin ZHANG, Yu LIU and Jiaru LIN, "A Dynamic Hysteresis-adjusting Algorithm in LTE Self-Organization Networks", Vehicular Technology Conference (VTC Spring), 2012 IEEE 75th IEEE, 2012.

[5] MIMO Transmission Schemes for LTE and HSPA Networks, 2009.

[6] Hao Wang, Lianghui Ding, Ping Wu, Zhiwen Pan, Nan Liu and Xiaohu You, "Dynamic Load Balancing and Throughput Optimization in 3GPP LTE Networks", Proceedings of the 6th International Wireless Communications and Mobile Computing Conference ACM 2010.

[7] WANG Min, FENG Chun-yan and ZHANG Tian-kui, "Min-max load balancing scheme for mixed multicast and unicast services in LTE networks", The Journal of China Universities of Posts and Telecommunications 19.2 (2012).

[8] LI Bo, WANG Xi-yuan and YANG Da-cheng, "Interdomain traffic balancing with co-channel interference management in multi-domain heterogeneous network for LTE-A", The Journal of China Universities of Posts and Telecommunications 2011.

[9] WANG Hao, LIU Nan, LI Zhihang, WU Ping, PAN Zhiwen1 \& YOU Xiaohu, "A Unified Algorithm for Mobility Load Balancing in 3GPP LTE Multi-Cell Networks", Science China Information Sciences 56.2 (2013).

[10] Ming Li, Liang Zhao, Xi Li, Xiaona Li, Yasir Zaki, Andreas Timm-Giel and Carmelita Görg, "Investigation of Network Virtualization and Load Balancing Techniques in LTE Networks", Vehicular Technology Conference (VTC Spring), 2012 IEEE 75th IEEE, 2012.

[11] Omar Altrad and Sami Muhaidat, "Load Balancing Based on Clustering Methods for LTE Networks", algorithms 8 (2013).

[12] Network Simulator: http:///www.isi.edu/nsnam/ns 